\title{
Migraine in young females with irritable bowel syndrome: still a challenge
}

This article was published in the following Dove Press journal:

Neuropsychiatric Disease and Treatment

\section{Doina Georgescu' \\ Daniela Reisz ${ }^{2}$ \\ Camelia Vidita Gurban ${ }^{3}$ \\ Liviu Andrei Georgescu ${ }^{4}$ \\ loana Ionita ${ }^{5}$ \\ Oana Elena Ancusa' \\ Daniel Lighezan' \\ 'Department of Internal Medicine, "Victor Babes" University of Medicine and Pharmacy, Timisoara, ${ }^{2}$ Department of Neurology, "Victor Babes" University of Medicine and Pharmacy, Timisoara, ${ }^{3}$ Department of Biochemistry and Pharmacology, "Victor Babes" University of Medicine and Pharmacy, Timisoara, ${ }^{4}$ Department of Urology, "Victor Babes" University of Medicine and Pharmacy, Timisoara, ${ }^{5}$ Department of Hematology, "Victor Babes" University of Medicine and Pharmacy, Timisoara, Romania}

Correspondence: Daniela Reisz Department of Neurology, "Victor Babes" University of Medicine and Pharmacy, Piata E. Murgu 2, Timisoara 30004I, Romania

Email reisz_daniela@yahoo.com

\begin{abstract}
Migraine without aura is frequently reported in female patients with irritable bowel syndrome (IBS), but knowledge about the relationship between these two conditions is still lacking. This study was aimed to explore the particularities of migraine without aura in young female patients with IBS in order to establish a possible link between them. From a cohort of young female patients hospitalized with IBS in the Internal Medicine Department, 30 joined this pilot study, and they were assigned into two groups on the basis of presence or absence of migraine. In this sample, 15 patients have mild to moderate migraine without aura, with a recently taken normal brain scan, and 15 were without migraine. Diseases and conditions not related to migraine and other possible specific female comorbidities were ruled out. Patients undertook a thorough clinical examination in order to assess fibromyalgia (FM) and chronic pelvic pain (CPP), Questionnaires for migraine disability assessment (MIDAS) and generalized anxiety disorder (GAD) were performed. Laboratory testing of blood, urine, and stool were also performed. Optimized lymphocyte proliferation test for food allergy (FA) and a fecal microbiota (microbiological semiquantitative method) for dysbiosis (DB) assessment were performed. Based on the results, migraine-positive group displayed more severe comorbidities: $\mathrm{FM}(p=0.0002)$, FA ( $p=0.0006), \mathrm{CPP}(p=0.026)$, higher scores of anxiety (GAD, $p=0.0008)$, and more severe $\mathrm{DB}(p=0.0009)$. We noticed a strong positive correlation between MIDAS and GAD $(r=0.83)$, a good positive correlation between MIDAS and DB $(r=0.56)$, and a moderate positive correlation between MIDAS, FM, and FA ( $r=0.46$ and 0.41 ). In conclusion, young female patients with IBS and migraine without aura displayed more severe associated issues - anxiety, intestinal DB, FM, FA, and CPP. The severity of migraine correlated well with anxiety range and DB magnitude and moderately with FM and FA.
\end{abstract}

Keywords: migraine without aura, IBS, young females

\section{Introduction}

Irritable bowel syndrome (IBS) continues to be an intriguing condition, both for the clinicians and for the experienced researchers. While we don't yet fully understand the exact pathophysiological pathways, the great variability and lack of specificity of symptoms, as well as the overlap with several conditions, make IBS still puzzling. ${ }^{1}$

IBS is known as a functional gastrointestinal (GI) disorder, characterized by abdominal pain and altered bowel habits, in the absence of a specific or unique organic pathology, very often present in young women. ${ }^{2}$

Pain, either abdominal or extra-abdominal, is one of the most common complaints shared by patients with IBS, frequently resulting in a diminished quality of life. Some authors explained IBS pain pathophysiology as three parts of complexity: altered GI motility, visceral hyperalgesia, and an abnormal processing of information by the central nervous system (CNS). Altered GI motility includes distinct aberrations in 
small and large bowel motilities and is probably associated with a generalized smooth muscle hyperresponsiveness. Visceral hyperalgesia characterizes IBS secondary to an enhanced perception of normal motility and rectosigmoid balloon inflation produces more pain at lower volumes in patients than in controls. Sensitization of the intestinal afferent nociceptive pathways, which synapse in the dorsal horn of the spinal cord, could provide a unifying mechanism for abdominal pain. ${ }^{3}$

Functional pain conditions like IBS, fibromyalgia (FM), chronic pelvic pain (CPP), migraine, and temporomandibular joint disorder share many similarities. Recent studies reported that people having GI conditions experienced headache more often, including migraine. Many migraineurs complained of digestive disturbances not only within migraine's attacks but also in intercritical periods. Migraine is frequently present in patients with IBS, as a comorbidity, sometimes along with other pain conditions of unknown etiology, resulting in increasing the burden of suffering and lowering the quality of life..$^{4-7}$

Some studies concluded that migraine headache or other types of headache (cluster headache) are more likely to be present in people already having GI disorders. ${ }^{8}$

Headache is frequently reported in clinical observation and epidemiological studies in people with IBS, often coexisting with FM and other chronic pain syndromes and functional conditions. A prevalence study showed that people in IBS cohorts had significantly higher odds to have comorbidities (40\%-80\%) like migraine, FM, and depression. ${ }^{9}$ Both disorders, migraine and IBS, are diagnosed to be more prevalent in young women. ${ }^{10}$

The aim of the study was to explore the particularities of migraine without aura in young female patients with IBS in order to establish a possible link between them.

\section{Patients and methods}

\section{Research participants' recruitment}

From a pool of young female patients (aged $<45$ years) hospitalized with different clinical types of IBS (constipation predominant [IBS-C], diarrhea predominant [IBS-D], or mixed [IBS-M]) at the Internal Medicine Department, 30 joined this pilot study, and they were assigned into two groups on the basis of the presence or absence of migraine: 15 having migraine without aura of mild to moderate severity, with a recently taken normal brain scan, and 15 without migraine. We used the current flat rules of thumb for overall pilot trial sample size. As recommended, the pilot trial sample size was $30 .{ }^{11,12}$ Patients with diabetes mellitus, obesity
$\left(\mathrm{BMI}>30 \mathrm{~kg} / \mathrm{m}^{2}\right)$, thyroid or collagen diseases, chronic organ failure (pulmonary, cardiac, or renal), cancer, associated inflammatory bowel disease, celiac disease or gluten sensitivity, ongoing or recent antibiotic treatment or use of probiotics, as well as smokers, were excluded.

The study was approved by the Ethical Board of the University of Medicine and Pharmacy "Victor Babes" from Timisoara and written informed consent was obtained from all the participants.

\section{Procedure and data recording}

Definition and diagnostic criteria of migraine changed over time reflecting the improved understanding of some disorders and the identification of new ones. ${ }^{13,14}$ The present study used the diagnostic criteria of migraine without aura, issued in 2010, by the Third British Association for the Study of Headache $^{15}$ :

Diagnostic criteria of migraine without aura are as follows:

- A: At least 5 attacks fulfilling criteria B-D;

- B: Headache attacks lasting 4-72 hours (untreated or unsuccessfully treated);

- $\mathrm{C}$ : Headache has at least two of the following characteristics: 1) unilateral location 2) pulsating quality (varying with the heartbeat) 3) moderate or severe pain intensity 4) aggravation by or causing avoidance of routine physical activity (example, walking or climbing stairs);

- D: During headache at least one of the following: 1) nausea and/or vomiting, 2) photophobia and phonophobia;

- E: Not attributed to another disorder (history and examination do not suggest a secondary headache disorder or, if they do, it is ruled out by appropriate investigations or headache attacks do not occur for the first time in close temporal relation to the other disorder).

The assessment of migraine without aura was made by a qualified neurologist, taking into account the possibility that symptoms of migraine could be nonspecific and, therefore, could be present in other primary or secondary headache conditions. ${ }^{16,17}$

Assessment of severity of the migraine attacks was set according to the migraine disability assessment (MIDAS) questionnaire. ${ }^{18}$ The MIDAS questionnaire was used in order to assess the disability related to the headache during the daily activities (work, home, and family commitments, leisure or social activities). The disability due to migraine was graded into four classes according to MIDAS scores: 0-5 as minimal, $8-10$ as mild, $11-20$ as moderate, and $>21$ as severe disability. 


\section{Definition and diagnostic criteria of IBS}

As recommended by Rome III consensus, ${ }^{19}$ IBS as a functional bowel disorder, has the following diagnostic criteria that has been fulfilled for the last 3 months with symptom onset at least 6 months prior to diagnosis: recurrent abdominal pain or discomfort (uncomfortable sensation not described as pain) at least 3 days/month in the last 3 months associated with two or more of the following: 1) improvement with defecation, 2) onset associated with a change in frequency of stool, and 3) onset associated with a change in form (appearance) of stool.

An FM diagnostic protocol was included as a special part of the clinical examination. Patients should meet three conditions: 1) widespread pain index (WPI) $\geq 7$ and symptom severity (SS) scale score $\geq 5$ or $3<$ WPI $<6$ and SS scale score $\geq 9$; 2) symptoms have been present at a similar level for at least 3 months; and 3) the patient does not have a disorder that would otherwise explain the pain. Ascertainment 1) WPI: note the number of areas in which the patient has had pain over the last week: shoulder girdle, left; hip (buttock, trochanter), left; jaw, left; upper back; shoulder girdle, right; hip (buttock, trochanter), right; jaw, right; lower back; upper arm, left; upper leg, left; chest; neck; upper arm, right; upper leg, right; abdomen; lower arm, left; lower leg, left; lower arm, right; lower leg, right. Score will be between 0 and 19. For each of the three symptoms above, the level of severity over the past week was indicated using the following scale: $0=$ no problem; $1=$ slight or mild problems, generally mild or intermittent; $2=$ moderate, considerable problems, often present and/or at a moderate level; $3=$ severe, continuous, life-disturbing problems. ${ }^{20}$

\section{CPP diagnosis and severity assessment}

CPP was defined as a functional regional hypersensitivity resulting in pelvic floor symptoms (urinary or anal distress), pain being continuous or recurrent, for at least 6 months. The scale of discomfort was set ranging from 0 to 3 : 0 , absent; 1 , mild; 2 , moderate; 3 , severe..$^{21}$ Other possible femalespecific comorbidities were ruled out.

\section{Generalized anxiety disorder (GAD) self-assessment of anxiety}

GAD self-assessment of anxiety score was used with 5, 10, and 15 cutoff points for different range of disturbances: under 5 , minor; 6-10, moderate; $11-21$, severe. ${ }^{22}$

\section{Food allergy (FA) tests}

FA tests as optimized lymphocyte proliferation test were also performed for 70 most frequent substances involved in alimentary allergic reactions in Central and East Europe ( $>95 \%$ of known fourth types of FAs). We noted 0 for a full negative test, 1 for single substance detected, 2 for two substances, 3 or more for as many as counted allergic compounds found. ${ }^{23}$

\section{Blood smear and biochemical exams}

Routine laboratory tests for erythrocyte sedimentation ratio (ESR), C reactive protein, tumor necrosis factor (TNF)-alpha, fasting blood sugar (glucose), alanine transaminases (ALT), creatinine, urine biochemical and bacteriological tests, and stool exams (calprotectin, Helicobacter pylori antigen, Giardia lamblia antigen, cytology, and fecal hemoccult) were performed.

The stool samples for the microbiological analysis were collected separately in special sterile containers. Each participant was briefed about the correct collection and transportation of the stool samples. The samples were brought to the laboratory as soon as possible. Processing of stool samples started at the maximum of $2 \mathrm{~h}$ time interval after collection. First, a progressive dilution in saline water from $10^{-1}$ to $10^{-5}$ was performed. Then, $0.1 \mathrm{~mL}$ of stool samples were placed on the surface of special plates containing different culture media. Microbiological examination was performed in order to determine aerobe, anaerobe, and microaerophiles spp. and to assess the colony forming units (CFU) per gram stool. ${ }^{24}$ The aerobe species (agar chromID and CPS) determined were Escherichia coli and E. coli variants, Proteus spp., Klebsiella spp., Enterobacter spp., Serratia spp., Morganella morganii, Citrobacter spp., Pseudomonas spp., Enterococcus spp., and Staphylococcus aureus. In order to detect the anaerobe species, plates were incubated at $37^{\circ} \mathrm{C}$ for $48 \mathrm{~h}$ on special culture media (anaerobe basal agar CM0972) under specific anaerobic microclimate conditions. CFU of Bacteroides spp., Bifidobacterium spp., Lactobacillus spp., Clostridium spp., Clostridium difficile, and all aerobic spp. were counted after Gram staining. Salmonella spp., Shigella spp., and Yersinia spp. were always checked out. In selected cases, toxins $\mathrm{A} / \mathrm{B}$ of $C$. difficile, Norwalk virus, enteroaggressive $E$. coli strains (EAggEC), enterotoxic E. coli (ETEC) strains, and enteroinvasive $E$. coli (EIEC) strains were also determined. Cultures for fungi Candida albicans and non-albicans and Geotrichum spp. were performed. After assessment of CFU for each species, increase or decrease of diverse microorganisms (+ modestly, ++ moderately, +++ greatly) was semiquantitatively reported. Borderline cases were also mentioned as (+) or (-). The microbiological chart of the stool ranged from physiological intestinal (normobiosis) 
to various degrees of dysbiosis (DB): 1, mild; 2 , moderate; and 3, severe.

\section{Statistical analysis}

GraphPad InStat 3 and GraphPad Prism 7 software (GraphPad Software, Inc., La Jolla, CA, USA) were used with the panel for continuous or categorical data. Descriptive statistic was run, and all mathematical numerical values were expressed as mean values and SD. The unpaired $t$-test was also used for statistical distribution and interpretation of $p$-values with $95 \%$ CI. The cutoff for statistical significance was $p \leq 0.05$. Calculation of chi-squared test was meant to compare the two groups when analyzed variables were categorical. Nonparametric Spearman correlation test was performed, with the assessment of $r$ coefficient, in order to state the magnitude and direction of correlations.

\section{Results}

Regarding the age of research participants, as seen in Table 1, there are no statistically significant differences between the two groups $(p=0.7964)$. However, the second group (migraine positive) displayed significant differences related to comorbidities, like FM, CPP, FA, and anxiety score (GAD; $p \leq 0.05)$. Alcohol and substance abuse were more frequently recorded in the second group (IBS with migraine), but not in a significant range: $13.3 \%$ vs $33.3 \%$ for alcohol abuse, $p=0.2027$ and $6.6 \%$ vs $20 \%$ for drug abuse, $p=0.2880$. There was no statistically significant difference between the two study groups, related to the clinical types of IBS as well.

Comorbidities are more often present and at a higher range of severity in the second group (migraine positive), as seen in Figure 1A and B. FM is reported in only $33.3 \%$ in the first group (migraine negative) compared with the second

Table I Clinical data flowchart in IBS patients

\begin{tabular}{llll}
\hline & Migraine negative & Migraine positive & $\boldsymbol{p}$-value \\
\hline Age (years) & $39.00 \pm 5.63$ & $39.47 \pm 4.05$ & $\mathbf{0 . 7 9 6 4}$ \\
CPP & $0.60 \pm 0.51$ & $1.27 \pm 0.59$ & $\mathbf{0 . 0 0 2 6}$ \\
FM & $0.28 \pm 0.50$ & $1.44 \pm 0.92$ & $\mathbf{0 . 0 0 0 2}$ \\
FA & $0.33 \pm 0.49$ & $1.27 \pm 0.80$ & $\mathbf{0 . 0 0 0 6}$ \\
GAD score & $4.20 \pm 1.26$ & $8.13 \pm 3.85$ & $\mathbf{0 . 0 0 0 8}$ \\
Alcohol abuse & $13.3 \%$ & $33.3 \%$ & 0.2027 \\
Drug abuse & $6.6 \%$ & $20 \%$ & 0.2880 \\
IBS-D & $33.3 \%$ & $40 \%$ & 0.7081 \\
IBS-C & $53.3 \%$ & $46.6 \%$ & 0.7182 \\
IBS-M & $13.3 \%$ & $13.3 \%$ & 1 \\
\hline
\end{tabular}

Notes: All numerical values are expressed as mean \pm SD unless indicated otherwise. $p$-values in bold represent statistically significant difference.

Abbreviations: CPP, chronic pelvic pain; FA, food allergy; FM, fibromyalgia; GAD, generalized anxiety disorder; IBS, irritable bowel syndrome; IBS-C, constipationpredominant IBS; IBS-D, diarrhea-predominant IBS; IBS-M, alternating constipation/ diarrhea (mixed) IBS. group (migraine positive), where almost all patients (80\%) had this complaint. The same condition was seen for CPP ( $40 \%$ vs $93.3 \%$ ). FAs were also more frequently found in the second group (migraine positive): $80 \%$ vs $33.3 \%$. DB was often seen in both groups, however, the second one (migraine positive) displayed more severe forms.

Figure 2 displays the severity of anxiety (GAD) in research participants, highlighting the fact that the second study group (migraine positive) encountered more severe forms, showing a statistically significant difference when compared with the first group (migraine negative), $p=0.0008$.

As shown in Table 2, no statistically significant differences were noted between our two groups concerning different biological data: ESR, hemoglobin (Hb), leucocytes (L), platelets (Plt), glucose (Gl), creatinine (creat), and transaminases (ALT). However, the study of fecal microbiota displayed a significant statistical difference between the two groups: the second group (migraine positive) presented a higher incidence and severity of DB.

As illustrated in Figure 3, fecal DB was significantly more frequent in the second group (migraine positive): $86.88 \%$ vs $40 \%(p=0.0088)$. All the patients from the first group (migraine negative) with fecal DB displayed only mild forms, whereas those from the second group (migraine positive) had significantly increased fecal DB ranges $(p=0.0009)$.

A study of MIDAS possible correlations was performed in the second group (migraine positive). As shown in Figure 4, MIDAS correlated most significantly with GAD scores $(r=0.83)$, showing a very strong correlation. A good, positive correlation was observed with DB $(r=0.56), \mathrm{FM}(r=0.46)$, and FA $(r=0.41)$ and a very weak correlation was seen with CPP $(r=0.03)$.

As depicted in Figure 5, we also observed a good correlation between DB, MIDAS, and GAD scores $(r=0.5)$ in the migraineurs group.

\section{Discussion}

Chronic headache, such as migraine, along with other extradigestive comorbidities such as FM, CPP, interstitial cystitis, insomnia, depression, and anxiety is reported to be frequently associated with IBS. ${ }^{25,26}$ Some authors found out that IBS subjects displayed a twofold increasing risk of somatic comorbidities, possibly in the context of an overlapping pathophysiological pathways; more than $65 \%$ of patients presented association to $\mathrm{FM}, \mathrm{CPP}$, and chronic fatigue syndrome. ${ }^{27}$ In our series, $80 \%$ of the migraine-positive patients experienced extradigestive comorbidities like FM, CPP, and anxiety. Are these conditions just coincidentally associated, or is there some link between? The hypothesis of 

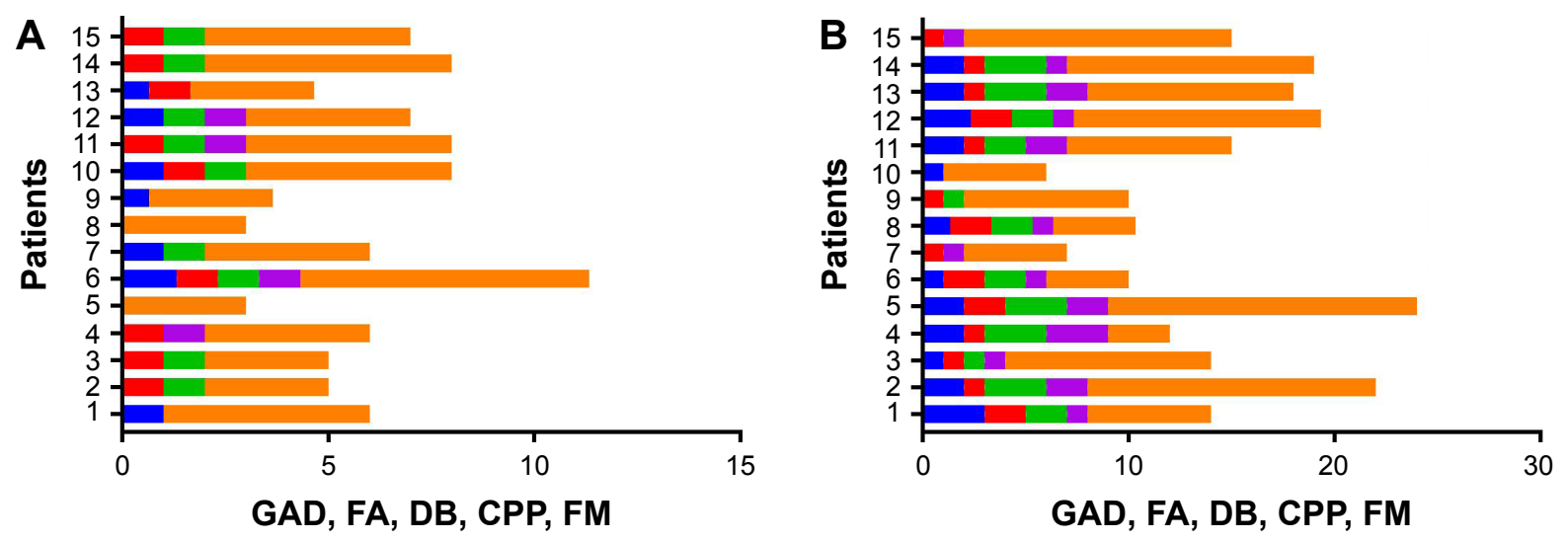

GAD FA DB $\square$ CPP $\square$ FM

Figure I Presence and severity of comorbidities: GAD, FA, DB, CPP, and FM in (A) migraine-negative group and (B) migraine-positive group. Abbreviations: CPP, chronic pelvic pain; DB, dysbiosis; FA, food allergy; FM, fibromyalgia; GAD, generalized anxiety disorder.

affective spectrum disorders (ASD) suggests that IBS, FM, migraine, and other psychiatric and medical disorders share some causality conditions, based on the clinical observation of responsiveness to the same chemical classes of antidepressants, possibly related to familial aggregation and genetic similarities. ${ }^{28}$ Many researches provided us with increasing evidence of functional pain syndrome (FPS), which associates IBS, migraine, FM, CPP, and many other conditions that share many genetic particularities, neuroimaging aspects, and some therapeutical response. Polymorphisms in the catechol-O-transferase gene seem to be associated with FPS, favoring the hypothesis of genetic involvement. Since these conditions show an important overlapping side, researchers move on and shift this model with that of a chronic sensitivity syndrome. In the end, all these functional complaints are a phenotypic expression of multiple interrelations between genetic predictors and other environmental factors that act as triggers..$^{29,30}$ Since hereditary aggregation

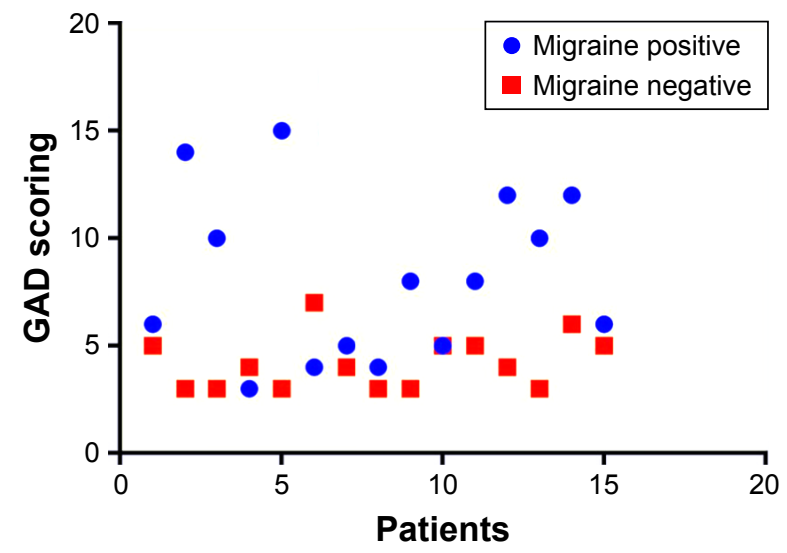

Figure 2 Severity of anxiety (GAD) in IBS research participants.

Abbreviations: GAD, generalized anxiety disorder; IBS, irritable bowel syndrome. and genetic particularities are difficult to manage yet, a practical approach of FPS, based on environmental factors study, could be a possible lead to a better understanding of this issue. Thus, we have focused our research on finding out answers to some common questions, and to our knowledge, this is the first study addressing this issue. Could stress and anxiety act as triggers for relapsing of IBS and migraine attacks as well? Last decades have shown evidence based on functional brain imaging and morphometric studies demonstrating an association between IBS, FM, and stress, with the increasing of the hypothalamic activity. An increased response to stimuli in the insula and anterior cingulate cortex was observed, revealing a possible way of braingut connection. ${ }^{31}$ Regarding migraine and mood disorders, many researchers focused more on depression, underrating the importance of anxiety. ${ }^{32,33}$ Quite contrarily, according to other studies, the prevalence of anxiety among migraineurs seemed to be higher than the prevalence of depression. ${ }^{34,35}$ High rates of anxiety have been reported among patients

Table 2 Biological flowchart in IBS research participants

\begin{tabular}{llll}
\hline & Migraine negative & Migraine positive & $p$-value \\
\hline $\mathrm{Hb}(\mathrm{g} / \mathrm{dL})$ & $12.58 \pm 0.8 \mathrm{I}$ & $12.44 \pm 0.75$ & 0.6287 \\
$\mathrm{~L} / \mathrm{mm}^{3}$ & $7.49 \times 10^{3} \pm 0.73 \times 10^{3}$ & $7.20 \times 10^{3} \pm 0.7 \times 10^{3}$ & 0.3725 \\
$\mathrm{Plt} / \mathrm{mm}^{3}$ & $257.33 \times 10^{3} \pm 55.25 \times 10^{3}$ & $276.33 \times 10^{3} \pm 73.1 \mathrm{I} \times 10^{3}$ & 0.4287 \\
$\mathrm{ALT}(\mathrm{IU} / \mathrm{L})$ & $29.67 \pm 1.44$ & $26.07 \pm 1.73$ & 0.1207 \\
Creat $(\mathrm{mg} / \mathrm{dL})$ & $0.76 \pm 0.09$ & $0.80 \pm 0.09$ & 0.25 \\
$\mathrm{Gl}(\mathrm{mg} / \mathrm{dL})$ & $87.13 \pm 7.22$ & $88.33 \pm 5.73$ & 0.6180 \\
$\mathrm{ESR}(\mathrm{mm} / \mathrm{h})$ & $9.73 \pm 3.08$ & $9.60 \pm 3.14$ & 0.9073 \\
$\mathrm{DB}$ & $0.60 \pm 0.5 \mathrm{I}$ & $1.8 \pm \mathrm{I} .15$ & $\mathbf{0 . 0 0 0 9}$ \\
\hline
\end{tabular}

Notes: All numerical values are expressed as mean \pm SD. $p$-value in bold represents statistically significant difference.

Abbreviations: ALT, alanine transaminases; Creat, creatinine; DB, dysbiosis; ESR, erythrocyte sedimentation ratio; $\mathrm{Gl}$, glucose; Hb, hemoglobin; IBS, irritable bowel syndrome; L, leucocytes; Plt, platelets. 


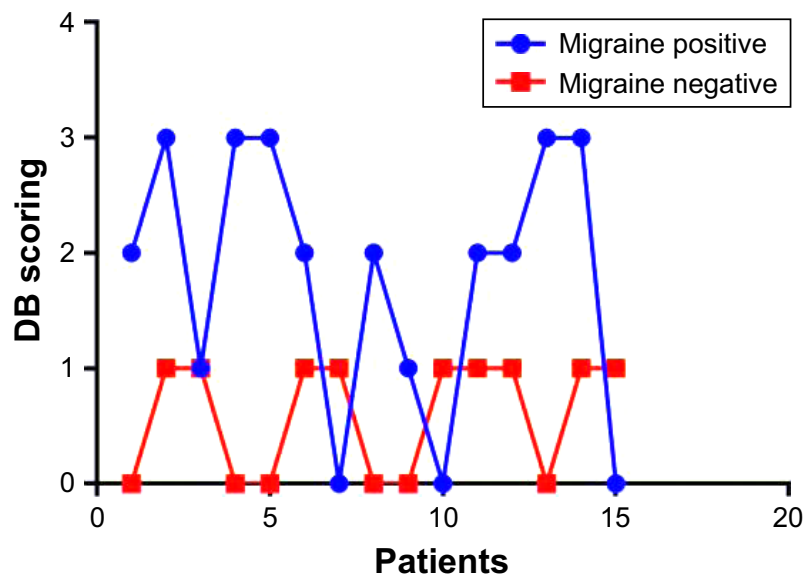

Figure 3 Study of DB severity in IBS patients. Abbreviations: DB, dysbiosis; IBS, irritable bowel syndrome.

with chronic pain syndrome, as well. ${ }^{36}$ A recent cohort Canadian study concluded that there is a robust association between anxiety disorder and migraine, where debilitating pain and limitation in instrumental activities of daily living could be mediators in this relationship. ${ }^{37}$ From the clinician perspective, a lot of flare-ups in both migraineurs and IBS patients are related to stress mismanagement and anxiety. Our results support these observations very well, suggesting a close connection between anxiety, IBS, and migraine, with a strong correlation between MIDAS and GAD scores. In this series, patients with IBS and associated migraine displayed not only higher rates of anxiety but also tended to be more exposed to alcohol and drug abuse. Intestinal microbiota and its genetic footprint have already been intensively studied. Many papers highlight DB implication not only in many local IBS complaints (abdominal pain, bloating and altered bowel habits) but also in modulation of enteral reactivity, signaling process, and dialogue in the

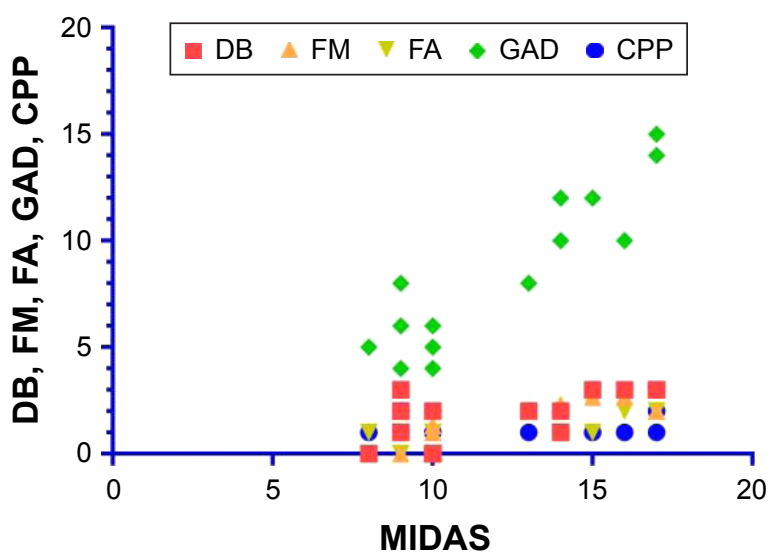

Figure 4 MIDAS correlations with DB, FM, FA, GAD, and CPP in migraine-positive patients.

Abbreviations: CPP, chronic pelvic pain; DB, dysbiosis; FA, food allergy; FM, fibromyalgia; GAD, generalized anxiety disorder; MIDAS, migraine disability.

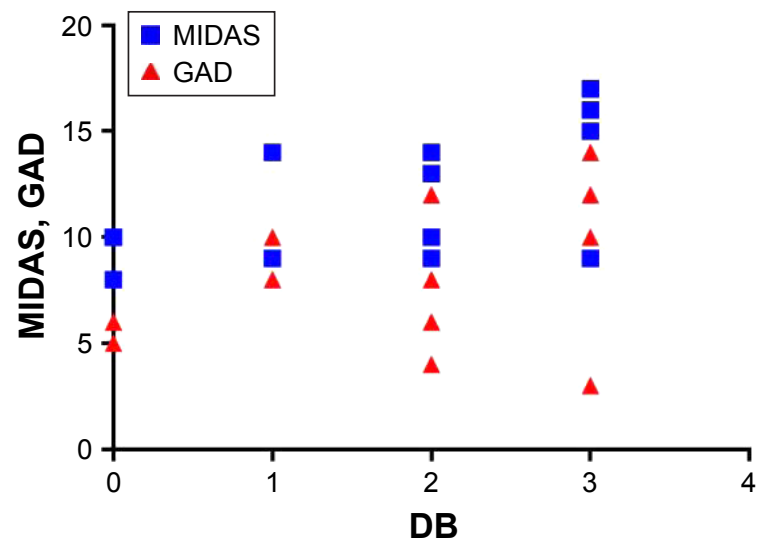

Figure 5 Fecal DB correlations: MIDAS and GAD in migraine-positive patients. Abbreviations: DB, dysbiosis; GAD, generalized anxiety disorder; MIDAS, migraine disability.

brain-gut connection. ${ }^{38}$ Is it possible for some patients to develop an alteration of enteral permeability, with changing of the local immune response, resulting in a modification of local microbiota? ${ }^{39}$ Since intestinal DB is involved in many pathophysiological pathways in IBS, could microbiota also play a role in migraine-associated comorbidity? It seems that gut-brain axis (GBA) goes bidirectional when it comes for microbiota: either via signaling channels from gut microbiota to CNS, or inversely, from CNS to enterocolonic microbiota via different communication pathways. In fact, there are complex interactions going both ways, IBS being a good example of a twisted cross talk between gut and brain. This communication system between gut and brain transcend the simple maintenance of digestive homeostasis, having far more implications regarding affectivity, motivation, and cognition, where complex mediators are involved. A recent prospective study has shown interesting evidence that independent gut to brain and brain to gut pathways operate in IBS and functional dyspepsia. After 1 year survey, they were able to demonstrate the odds of anxiety development in patients with IBS and conversely the odds of IBS development in patients with anxiety. ${ }^{40,41}$ There are studies observing a higher level of circulating mediators like cytokines, including TNF-alpha, suggesting a possible low grade of gut inflammation, with involvement of eosinophils and mast cells, which could explain several neuropsychological comorbidities associated to IBS. .2, $^{43}$ Thus, GBA acts as a network program, involving many parties, including autonomic and enteric nervous system, also hypothalamic-pituitary-adrenal (HPA) axis, integrating and monitoring a lot of data in order to realize a complex communication with different channel drives and multiple functions. ${ }^{44,45}$ In this view, our results showed a good positive correlation between MIDAS and DB severity, hypothesizing 
the fact that microbiota disruption could also play a role (either pathogenic or as a cofactor) in associated migraine in IBS patients.

Food intolerance or allergy can sometimes trigger migraine attacks as well as IBS relapse. In some cases, there are immediate attacks after ingestion of some food containing nitric oxide mediators, that act as vasodilators, while others experience more delayed attacks, like migraines, related to releasing of specific peptides or immune response. ${ }^{46,47}$ The present study reported a good positive correlation between severity of migraine (MIDAS) and FA, opening an interesting discussion about the possible implications of dietary triggers. Will using low fermentable oligosaccharides disaccharides monosaccharides and polyols (FODMAP), via microbiome changing metabolism, also results in alleviating the migraine severity along with symptoms characterizing IBS ${ }^{48}$

Alterations in HPA axis regulation in response to stress, resulting in behavioral and peripheral changes, could trigger the onset of different diseases or disease progression, like depression/anxiety disorders, hyperalgesia, FM, and chronic fatigue syndrome. ${ }^{49}$ Some clinical evidence support the hypothesis of gut microbiota implication via GBA program in depression/anxiety disorders, as well. ${ }^{50}$ It is yet not so easy to draw a clear line in the relationship between the two issues migraine and IBS: either a simple conjoint association or really interdependent. ${ }^{51}$ Our results revealed good positive correlations between microbiota (DB range) and severity of anxiety (GAD) in patients having IBS and associated migraine attacks. Thus, microbiota seems to be bidirectionally implicated in this GBA complex program; it looks that its dysregulation could intervene not only in the aggravation of IBS status but also in the setting of migraine severity. From this point, there is just a short step to the next question: is it possible to adjust the medical treatment and prevent migraine attack in patients with IBS by simply using probiotics? A recent randomized, double-blind, placebo-controlled pilot study that investigated the effects of probiotics on anxiety/ depression in patients with IBS concluded that after 6 weeks of daily consumption, there were good results with reduction in depression scores and decrease in limbic reactivity in the brain. ${ }^{52}$ Many hypotheses related to this challenging issue remain subject to debate for future research.

\section{Limitations}

A limitation of the present study could be related to the small number of the research participants. Selection criteria concerning enrollment of non-severe forms of migraine could also be a limitation. Further studies, in larger cohorts, approaching more severe forms of migraine, should determine the validity of these results, which seemed to us to be very representative and well correlated.

\section{Conclusion}

Young female patients having migraine without aura, associated with IBS, displayed several other comorbidities: anxiety, intestinal DB, FAs, FM, and CPP at a higher range of severity. In this series, the severity of migraine was positively well correlated with anxiety and DB range and moderately correlated with FM and FAs.

\section{Author contributions}

DG contributed to the conception and study design, drafting and critically revising the manuscript, also carrying out the analysis and interpretation of the data. DR, LAG, and CVG contributed to the acquisition, interpretation, and statistical analysis of the data. II, OEA and DL assisted in the editing and proofreading of the manuscript and reviewing of all tables and figures. All authors contributed in revising this manuscript and in carrying out the interpretation of the statistical analysis of the data. In addition, all authors hold rights to the intellectual content of this article.

\section{Disclosure}

The authors report no conflicts of interest in this work.

\section{References}

1. Camilleri M. Treating irritable bowel syndrome: overview, perspective and future therapies. Br J Pharmacol. 2004;141(8):1237-1248.

2. Chang L, Heitkemper MM. Gender differences in irritable bowel syndrome. Gastroenterology. 2002;123(5):1686-1701.

3. Camilleri M, Spiller R. Irritable Bowel Syndrome: Diagnosis and Treatment. Edinburgh: W.B. Saunders; 2002.

4. Adams K, Osmundsen B, Gregory WT. Does fibromyalgia influence symptoms bother from pelvic organ prolapsed? Int J Urogynecol. 2014; 25(5):677-682.

5. Davis JA, Robinson RL, Le TK, et al. Incidence and impact of pain conditions and comorbid illnesses. J Pain Res. 2011;4:331-345.

6. Malone CD, Bhowmick A, Wachholtz AB. Migraine: treatments, comorbidities, and quality of life, in the USA. J Pain Res. 2015;8:537-554.

7. Aamodt A, Stovner L, Hagen K, et al. Comorbidity of headache and gastrointestinal complaints. The head-HUNT study. Cephalalgia. 2008; 28(2):144-151.

8. Mulak A, Paradowski L. Migraine and irritable bowel syndrome. Neurol Neurochir Pol. 2005;39(4 Suppl 1):S55-S60.

9. Cole JA, Rothman KJ, Cabral HG, et al. Migraine, fibromyalgia and depression among people with IBS: a prevalence study. $B M C$ Gastroenterol. 2006;28(1):26.

10. Lipton RB, Stewart WF, Diamond S, et al. Prevalence and burden of migraine in the USA. Headache. 2001;41(7):646-657.

11. Kieser M, Wassmer G. On the use of upper confidence limit for the variance from a pilot sample for sample size determination. Biom J. 1996;38(8):941-949.

12. Julious SA. Sample size of 12 per group rule of thumb for a pilot study. Pharmaceut Stat. 2005;4(4):287-291.

13. Olesen J, Lipton RB. Migraine classification and diagnosis. International Headache Society criteria. Neurology. 1994;44(6 Suppl 4):S6-S10.

14. Lipton RB, Bigal ME, Steiner TJ, et al. Classification of primary headaches. Neurology. 2004;63(3):427-435. 
15. Samaan Z, MacGregor EA, Andrew D, et al. Diagnosing migraine in research and clinical settings: the validation of the Structured Migraine Interview (SMI). BMC Neurol. 2010;10(1):7.

16. Swanson JW, Sakai F. Diagnosis and differential diagnosis in migraine. In: Olesen J, Goadsby PJ, Ramadan NM, et al, editors. The Headaches. Philadelphia, PA: Lippincott Williams \& Wilkins; 2006:423-428.

17. Evans RW. Migraine mimics. Headache. 2015;55(2):313-322.

18. Stewart WF, Lipton RB, Dowson AJ, et al. Development and testing of the migraine disability assessment (MIDAS) questionnaire to assess headache related disability. Neurology. 2001;56(6 Suppl 1):S20-S28.

19. Drossman DA. The functional gastrointestinal disorders and the Rome III process. Gastroenterology. 2006;130(5):1377-1390.

20. Wolfe F, Claw DJ, Fitzcharles MA, et al. The American College of Rheumatology preliminary diagnostic criteria for fibromyalgia and measurement of symptom severity. Arthritis Care Res. 2010;69(5): 600-610.

21. Guideline American College of Obstetricians and Gynecologists (ACOG). ACOG practice bulletin; no. 51. Chronic pelvic pain. Obstet Gynecol. 2004;103(3):589-605.

22. Spitzer RL, Kroenke K, Williams JBW, Lowe B. A brief measure for assessing generalized anxiety disorder. Arch Intern Med. 2006; 166(10):1092-1097.

23. Janetschek P, Mayer W, Baehr V, et al. Diagnosis in vitro using an optimized lymphocyte proliferation test. Eur J Med Res. 2001; 4(6 Suppl I):75.

24. Brown R, Poxton IR, Wilkinson JF. Centrifuges, colorimeters and bacterial counts. In: Collee JG, Duguid JP, Fraser AG, Marmion BP, editors. Mackie and McCartney Practical Medical Microbiology, 14th ed. Edinburgh: Churchill Livingstone; 1999:200-247.

25. Hobson AR, Aziz Q. Brain imaging and functional gastrointestinal disorders: has it helped our understanding? Gut. 2004;53(8):1198-1206.

26. Yaguez L, Coen S, Gregory LJ, et al. Brain response to visceral aversive conditioning: a functional magnetic resonance imaging study. Gastroenterology. 2005;128(7):1819-1829.

27. Riedl A, Schmidtmann M, Stengel A, et al. Somatic comorbidities of irritable bowel syndrome: a systematic analysis. $J$ Psychosom Res. 2008;64(6):573-582.

28. Hudson JJ, Mangweth B, Pope GH, et al. Family study of affective spectrum disorder. Archiv Gen Psychiatry. 2003;60(2):170-177.

29. Wessely S, Nimnuan C, Sharpe M. Functional somatic syndromes: one or many? Lancet. 1999;354(9182):936-939.

30. Crabtree D, Ganty P. Common functional pain syndromes. BJA Educ. 2016;16(10):334-340.

31. Drossman DA. Brain imaging and its implications for studying centrally targeted treatments in irritable bowel syndrome: a primer for gastroenterologists. Gut. 2005;54(5):569-573.

32. Hamelsky SW, Lipton RB. Psychiatric comorbidities of migraine. Headache. 2006;46(9):1327-1333.

33. Buse DC, Silberstein SD, Manack AN, et al. Psychiatric comorbidities in episodic and chronic migraine. J Neurol. 2013;260(8):1960-1969.

34. Lanteri-Minet M, Radat F, Chautard MH, et al. Anxiety and depression associated with migraine: influence on migraine subjects disability and quality of life, and acute migraine management. Pain. 2005;118(3): 319-326.
35. Smitherman TA, Kolvas ED, Bailey JR. Panic disorder and migraine: comorbidity, mechanisms and clinical implications. Headache. 2012; 53(1):23-45.

36. Castro M, Kraychete D, Dalto C, et al. Comorbid anxiety and depression disorders in patients with chronic pain. Arq Neuropsiquiatr. 2009;67(4):982-985.

37. Fuller-Thomson E, Jayanthikumar J, Agbeyaka SK. Untangling the association between migraine, pain and anxiety: examining migraine and generalized anxiety disorder in a Canadian population-vase study. Headache. 2017;57(3):375-390.

38. Camilleri M, Di Lorenzo C. The brain-gut axis: from basic understanding to treatment of irritable bowel syndrome and related disorders. J Pediatr Gastroenterol Nutr. 2012;54(4):446-453.

39. Dupont HL. Evidence for the role of gut microbiota in irritable bowel syndrome and its potential influence on therapeutic targets. Aliment Pharmacol Ther. 2014;39(10):1033-1042.

40. Quigly EM. Bugs in the brain; brain the gut-seeking explanations for common gastrointestinal symptoms. Ir J Med Sci. 2013;182(1):1-6.

41. Koloski NA, Jones M, Tailey NJ. Evidence that independent gut to brain and brain to gut pathways operate in the irritable bowel syndrome and functional dyspepsia. Aliment Pharmacol Ther. 2016;44(6):592-600.

42. Keely S, Walker MM, Mark E, et al. Immune dysregulation in the functional gastrointestinal disorders. Eur J Clin Invest. 2015;45(12): $1350-1359$.

43. Locke JR, Weaver AL, Melton LJ, et al. Psychosocial factors are linked to functional gastrointestinal disorders: a population based nested casecontrol study. Am J Gastroenterol. 2004;99(2):350-357.

44. Rhee SH, Pothoukalis C, Mayer EA. Principles and clinical implications o the brain-gut-enteric microbiota axis. Nat Rev Gastroenterol Hepatol. 2009;6(5):306-314.

45. Carabotti M, Scirocco A, Masselli MA, et al. The gut-brain axis: interactions between enteric microbiota, central and enteric nervous system. Ann Gastroenterol. 2015;28(2):203-209.

46. Alpay K, Ertas M, Orhan EK, et al. Diet restriction in migraine, based on IgG against foods: a clinical double-blind, randomized, cross-over trial. Cephalalgia. 2010;30(7):829-837.

47. Gonzalez A, Hyde E, Sangwan N, et al. Migraines are correlated with higher levels of nitrate-, nitrite-, and nitric oxide-reducing oral microbes in the American Gut Project Cohort. mSystems. 2016;1(5): e00105-e000116.

48. Gibson PR, Shepherd SJ. Evidence-based dietary management of functional gastrointestinal disorders: the FODMAP approach. $J$ Gastroenterol Hepatol. 2010;25(2):252-258.

49. Tsigos C, Chroussos GP. Hypothalamic pituitary, neuroendocrine factors adrenal axis and stress. J Psychosom Res. 2002;53(4):865-871.

50. Naseribafrouei A, Hestad H, Avershina E, et al. Correlation between human fecal microbiota and depression. Neurogastroenterol Motil. 2014;26(8):1155-1162.

51. Chang FY, Lu CL. Irritable bowel syndrome and migraine: bystanders or partners? J Neurogastroenterol Motil. 2013;19(3):301-311.

52. Pinto-Sanchez MI, Hall GB, Ghajar K, et al. Probiotic Bifidobacterium longum $\mathrm{NCC} 3001$ reduces depression scores and alters brain activity: a pilot study in patients with irritable bowel syndrome. Gastroenterology. 2017;153(2):448-459.

Neuropsychiatric Disease and Treatment

\section{Publish your work in this journal}

Neuropsychiatric Disease and Treatment is an international, peerreviewed journal of clinical therapeutics and pharmacology focusing on concise rapid reporting of clinical or pre-clinical studies on a range of neuropsychiatric and neurological disorders. This journal is indexed on PubMed Central, the 'PsycINFO' database and CAS,

and is the official journal of The International Neuropsychiatric Association (INA). The manuscript management system is completely online and includes a very quick and fair peer-review system, which is all easy to use. Visit http://www.dovepress.com/testimonials.php to read real quotes from published authors. 